\title{
Modelo de estructura organizacional para los institutos universitarios, vinculante con la realidad socioeconómica venezolana
}

\author{
Model of Organizational Structure for University Institutes Binding with the Venezuelan \\ Socioeconomic Reality
}

\author{
Rafael Pertuz Belloso' \\ Colegio Universitario Monseñor de Talavera \\ Maracaibo, Venezuela \\ rafaelpertuz69@gmail.com
}

Recibido 13 de enero de 2013 • Corregido 18 de marzo de 2013 • Aceptado 11 de setiembre de 2013

Resumen. La presente investigación tuvo como objetivo proponer un modelo de estructura organizacional para los institutos universitarios, vinculante con la realidad socio económica venezolana. El estudio corresponde a una investigación de campo de carácter descriptiva, con un diseño no experimental trasversal. La población del estudio estuvo constituida por un conjunto de 746 profesores y directivos de los institutos tecnológicos de Cabimas y Maracaibo. La técnica de recolección empleada fue la encuesta con un cuestionario compuesto por 54 ítems. La técnica de análisis de los datos fue la distribución de frecuencias porcentuales. Los resultados obtenidos indican qué subsistemas no se encuentran integrados en las instituciones estudiadas, así como la coexistencia de tipologías estructurales burocráticas, además de una clara descontextualización en la aplicación de los planes de la nación, lo cual evidencia escasa pertinencia y vinculación con la realidad socio económica venezolana. Para solucionar esta realidad, se diseñó un modelo de estructura organizacional mixto departamental/matricial que integra el departamento en una red matricial que vincula la docencia, la investigación y la extensión. Para la aplicación de este modelo se propuso su instrumentación en tres etapas o fases que permitirán alcanzar las características operativas del modelo departamental.

Palabras claves. Modelo, estructura organizacional, realidad socio económica, institutos universitarios.

1 Doctor en Ciencias de la Educación, egresado de Universidad Dr. Rafael Belloso Chacín (URBE). Docente e investigador de las instituciones: Colegio Universitario Monseñor de Talavera e Instituto Universitario de Tecnología Pedro Emilio Coll. 


\begin{abstract}
The present study is aimed at proposing a model of organizational structure for university institutes binding with the Venezuelan socioeconomic reality. This is a descriptive nonexperimental cross-sectional research study. The study population included 746 professors and administration from the Cabimas and Maracaibo Technological Universities. Data was collected using a questionnaire consisting of 54 items and analyzed using the percentage frequency distribution. Results obtained indicate the sub-systems not integrated in the studied institutions, coexisting bureaucratic structural typologies, and a clear decontextualized implementation of the nation's plans, which shows low relevance and relationship to the Venezuelan socioeconomic reality. To remedy this situation, a mixed departmental/matrix organizational structure model was designed that integrates the department into a matrix network linking teaching, research, and social action projects. The implementation of this model was proposed in three stages or phases in order to achieve the operational characteristics of the departmental model.
\end{abstract}

Keywords. Model, organizational structure, socioeconomic reality, university institutes.

En la actualidad, la educación superior Venezolana se encuentra inmersa en un proceso de trasformación de sus estructuras organizacionales, permitiendo así el establecimiento de un intenso debate acerca de su pertinencia, calidad, municipalización, desarrollo científico e investigativo, planes de formación permanentes, modelos y enfoques educativos, entre otros, que permitan generar los cambios acordes con la realidad socioeconómica venezolana.

Tomando en cuenta estas consideraciones, el presente artículo forma parte de un proyecto de investigación realizado en el marco de la Maestría en Gerencia Educativa, donde se analizaron diferentes variables relativas a la propuesta de un modelo de estructura organizacional para los institutos universitarios de tecnología venezolanos. De los objetivos de este proyecto también se derivaron otros artículos (Pertuz, 2013), con los que el texto actual comparte algunos detalles metodológicos, principalmente, pues en el que ocupa estas páginas se aborda, de manera más amplia, el desarrollo de la propuesta.

Para Castellano (2002), "entre 1980 y 1998, ... el Estado venezolano se debilitó en su papel rector de la Educación Superior, generándose [sic] un proceso de deterioro de las plantas físicas, laboratorios, ... equipos y en general de las condiciones académicas de estas instituciones, entre las cuales las más afectadas fueron los institutos universitarios tecnológicos" (p. 1).

La autora menciona que desde el año 1999, el Ministerio de Educación declara la reorganización académica y administrativa de los institutos y colegios universitarios del país, para lo cual nombró Comisiones de Transformación y Modernización, encargadas de adoptar medidas administrativas y académicas que resulten procedentes en virtud de su competencia, y proyectar las reformas técnicas y administrativas que sean convenientes para la institución y elevarlas a la consideración del viceministro de políticas académicas.

La autora indica que la causa principal de la reorganización antes señalada se debió a que los institutos y colegios universitarios fueron perdiendo, paulatinamente, los principios 
filosóficos que los identificaban y se convirtieron en terrenos moldeables y altamente fértiles para la perpetuación del clientelismo político, además de la improvisación promovida por las cúpulas partidistas.

De esta manera, Castellano (2002) afirma que los institutos tecnológicos del país se encuentran divorciados de los postulados que le otorgan sentido y coherencia a la formación tecnocientífica, lo cual genera vicios dentro de su estructura organizacional, que la convierten en antagónica a los nuevos paradigmas gerenciales del siglo XXI, los cuales, exigen cambios radícales a corto plazo que permitan construir el camino para redefinir el modelo educativo imperante en respuesta a las demandas sociales y económicas.

Tomando en cuenta estos planteamientos, la problemática de la estructura organizacional resulta particularmente significativa, debido a los obstáculos organizacionales que se presentan para implementar estructuras innovadoras, ya que deben superar factores socioculturales muy enraizados en la actividades académico-administrativas que forman parte de las instituciones de educación superior, en especial el caracterizado en esta investigación, como son los institutos universitarios de tecnología.

En consecuencia, se presenta esta investigación con el fin de suministrar respuestas que aspiren a proponer un modelo de estructura organizacional para los institutos universitarios que sea vinculante con la realidad socioeconómica venezolana, a fin de lograr sincronía con la realidad socioeconómica que exige la nación.

Desde esta perspectiva, para Pertuz (2013), la estructura organizacional configura la forma como las personas se comportarán o como actuarán en el escenario organizacional, lo cual indica que los institutos universitarios de tecnología deben ser estudiados como organizaciones conformadas por agrupamientos humanos que persiguen objetivos comunes, en forma racional y consciente, y que a su vez están sujetas a una serie de restricciones y a determinados recursos, por lo cual toda tiene propiedades o características que poseen otras organizaciones, sin embargo, cada una de ellas con una serie exclusiva de esas características y propiedades.

De este modo, se debe asumir la estructura organizacional como un elemento integrador de las actividades desarrolladas en una organización, lo que la convierte en un instrumento a través del cual la organización puede lograr sus objetivos, así como también en instrumento utilizado por los mandos administrativos para definir las actividades por desarrollar, las personas que deban realizarlas, los recursos, las relaciones entre las personas, los puestos de trabajo, los sistemas que la integran y los procedimientos para el mejor desarrollo de las actividades académico-administrativas.

En vista de estos argumentos, se presenta esta propuesta como resultado de una investigación, que tuvo por como objeto de estudio la estructura organizacional y su vinculación con la realidad socioeconómica. Para obtener la propuesta de modelo de estructura organizacional, se diseñó una metodología inspirada en las experiencias de algunos 
autores utilizados en el estudio y ajustada al contexto de la educación superior venezolana, específicamente a las condiciones particulares de los institutos universitarios, en los que por primera vez se aplica un proceso de cambio estructural.

Ahora bien, el asunto cardinal de esta propuesta para los institutos tecnológicos estaría orientado a definir, en amplio sentido, su pertinencia socioeconómica, social e institucional, contenida en los planes de la nación, y establecer las orientaciones que permitan su acoplamiento orgánico con todas las instituciones que conforman el subsistema de la educación superior.

Finalmente, el presente estudio pretende propiciar, en forma definitiva, un cambio en la estructura organizacional que opera en estas instituciones, que conlleve a la solución de los problemas en una forma integral, con el mejor aprovechamiento de los recursos, y tratando de adecuar su pertinencia en la formulación de directrices y lineamientos para la solución de situaciones gerenciales relacionadas con la estructura académica-administrativa de dichas instituciones.

\section{Marco teórico}

La estructura es la forma en que una organización establece las relaciones entre sus componentes y que se refleja en el organigrama. Este es la representación gráfica del conjunto de actividades y procesos subyacentes en una organización. Existen tres componentes claves en la estructura (Daft, 2000, p. 34):

1. La designación de las relaciones formales, incluyendo el número de niveles en la jerarquía así como los tramos de control entre directores y supervisores.

2. La identificación del agrupamiento de los individuos por departamentos, áreas o secciones y de éstos en la organización total.

3. El diseño de sistemas para asegurar la comunicación, coordinación e integración efectivas de esfuerzos en todos los departamentos o áreas.

Estos tres elementos de la estructura corresponden tanto a los aspectos verticales como a los horizontales. Los dos primeros son el marco de referencia estructural, que es la jerarquía vertical que aparece en el organigrama. El tercero corresponde al patrón de interacciones entre los empleados y empleadas.

Considerando lo anterior, Brull y Gil (2005) presentan siete configuraciones estructurales, donde en cada una de ellas domina uno de los cinco mecanismos de coordinación, además consideran el papel de máxima importancia de cada una de las partes de la organización y utilizan un determinado tipo de descentralización: 
1. Organización empresarial o estructura simple: La estructura es sencilla y parecida a una gran unidad que consiste en uno (o pocos) gerentes ejecutivos que dominan y un grupo de operarios que hacen el trabajo básico....

2. Organización maquinal o burocracia maquinal: ... Los puestos se convirtieron en altamente especializados y el trabajo muy estandarizado. Primero requiere que la propia organización elabore su administración con una gran Tecnoestructura que diseña sus sistemas de estandarización.

3. Organización profesional o burocracia profesional: En esta organización predomina el impulso hacia la profesionalización. Al tener que depender de profesionales capacitados, la organización ha de hacer cesión de su poder y la estructura resultante es horizontal y altamente descentralizada. Existe poca necesidad de tecnoestructura ya que la principal estandarización sucede como resultado de la capacitación que se lleva a cabo fuera de la organización. ...

4. Organización diversificada o forma divisional: Es parecida a la profesional, pero en lugar de individuos está formada por unidades de línea intermedia, comúnmente denominadas divisiones. Cada división tiene su propia estructura. La divisionalización se hace porque las líneas de sus productos son diversificadas y eso se produce cuando se trata de organizaciones grandes y viejas. ...

5. Organización innovadora o adhocracia: La Adhocracia es una estructura orgánica que utiliza la adaptación mutua y los dispositivos de enlace. ...

6. Organización misionera: Cuando una organización es dominada por su ideología, sus miembros son estimulados a permanecer unidos $\mathrm{y}$, por consiguiente, tienen tendencia a tener una división del trabajo difusa, con poca especialización en sus puestos. Aquello que mantiene unida a la organización es la normalización de las normas, que los miembros compartan los mismos valores y creencias....

7. Organización política: ... No tiene ningún aspecto que predomine, a excepción del poder. Cuando una organización no tiene una parte que predomine, ningún mecanismo de coordinación que sobresalga y ninguna forma estable de centralización, es probable que tenga dificultades ajustando los conflictos internos. Aquello que caracteriza su comportamiento es el impulso a la desunión. (pp. 14-15)

Otro autor que considera los tipos de estructura organizacional es Toribio (1999), quien menciona la estructura matricial, la cual "responde a dos o más necesidades organizacionales. En la actividad universitaria, obedece a dos necesidades principales: la de especializar a las unidades funcionales que reúnen experiencia técnica y la de disponer de unidades que integren en programas y proyectos las actividades de docencia e investigación de esas unidades especializadas" (p. 10). Por otro lado, Pertuz (2013, p. 9) cita a Cardoso (2006, p. 52), quien plantea: 
URL: http://www.una.ac.cr/educare

CORREO: educare@una.cr

Que el enfoque de sistemas considera a la organización como una entidad que se encuentra en constante interacción con su ambiente. También señala la existencia de diversas relaciones entre sus componentes internos llamados subsistemas. Así, esta teoría conceptualiza a la organización como un sistema o un todo unitario compuesto por dos o más subsistemas interdependientes con ciertos límites vinculados a un suprasistema que es el medio ambiente.

Pertuz (2013) señala que:

Con base en lo anterior, puede deducirse, que esta teoría se presenta una diferencia sustancial con la teoría de la organización tradicional, ya que ésta utiliza un enfoque de sistema cerrado altamente estructurado; mientras que la de sistemas, la conceptualiza como una entidad abierta hacia los requerimientos del ambiente. En este aspecto, puede decirse que tipológicamente las estructuras organizacionales presentes actualmente en las IES venezolanas, suelen comportarse con una conceptualización o esquema tradicional, ya que estas suelen tener comportamientos inclinados hacia una entidad cerrada, sin embargo existen instituciones que contrariamente buscan relacionarse con el ambiente. (p. 9)

Siguiendo lo anterior, Anzaldo (1989) menciona que, para el análisis de la organización, se debe partir de diversos planteamientos provenientes de la teoría de organización. Tomando en cuenta este criterio, el autor conceptualiza la organización "como una entidad con límites relativamente identificables integrada por personas y órganos interrelacionados; con un cuerpo normativo; con sistemas de comunicación; con recursos humanos, materiales, económicos y tecnológicos; que existe en un medio ambiente para cumplir con fines específicos" (p. 1).

Asumiendo estos planteamientos, el autor conceptualiza la organización como un sistema total, que debe ser analizado desde el punto de académico-administrativo. Este se entiende como la manera, estrategia o modelo de gestión que cada institución adopta para la realización de sus funciones sustantivas. A partir de la noción de la organización de las IES como un sistema total se debe descomponer para su análisis, en cinco subsistemas básicos:

a) Subsistema ideológico: Caracterizado por aquellos elementos simbólicos que determinan las conductas tanto individuales como grupales.

b) Subsistema orgánico-estructural: Comprende lo referente a la infraestructura organizacional, así como la manera como esta ha sido acomodada.

c) Subsistema tecnológico: Comprende todos los elementos relativos a los procesos instrumentales por parte de la organización. 
d) Subsistema psicosocial: Hace referencia a las relaciones interpersonales en la organización tanto a nivel grupal como individual.

e) Subsistema normativo: conceptualiza aquellos preceptos y reglamentaciones que pretenden normar la actividad misma de la institució (Pertuz, 2013).

Para este autor, el análisis de estos subsistemas permite establecer diferenciaciones en cuanto al tipo de estructura organizacional de cualquier institución de educación superior; establecer la integración de estos subsistemas, a fin de proponer cambios concretos en la dinámica organizacional, además de sugerencias para que esta estructura organizacional adquiera dinamismo y eficacia; que se evite la repetición de los defectos e insuficiencias que han caracterizado a las instituciones de educación superior, cuya rigidez y desarticulación limita su eficacia y su alcance, así como su capacidad de enfrentarse con éxito a los procesos de cambio económico y social.

En otro orden de ideas, Gerring (1977), citado por Espinoza (2009, p. 15), plantea "que la planificación y gestión de un proceso de desarrollo económico-social, el cual es un componente de un sistema institucional de gobierno que se conforma como una estructura configurada por unidades funcionales, procesos técnicos, decisiones políticas, decisiones financieras, cosmovisión de sociedad; es un medio para vehiculizar la ideología política del gobierno nacional, así como la del gobierno regional y del gobierno local".

Desde esta perspectiva, la realidad social es asumida por todos aquellos aspectos ideológicos que formen parte de la construcción de cualquier proceso de planeación que se integre a la cosmovisión compartida o no por los integrantes de la sociedad. Desde esta mirada, se diseñan planes de desarrollo institucionales que plasmen la realidad socioeconómica que la ideología del gobierno de turno asuma para desarrollar ese modo de pensar específico.

Tomando en cuenta estos planteamientos, se considera que el Plan de Desarrollo Económico y Social de la Nación 2001-2007 constituye un sistema histórico-social que a través de la planificación legitima concepciones, teorías, conceptos y prácticas cuyos efectos contribuyen a la reproducción de la naturaleza de las relaciones sociales, que pueden traducirse en la realidad socioeconómica del país.

En este aspecto, es pertinente plantear que la planificación y gestión de un proceso de desarrollo económico-social es un medio para vehiculizar la ideología política del gobierno nacional, así como la del gobierno regional y del gobierno local, donde la realidad social es asumida por todos aquellos aspectos ideológicos que formen parte de la construcción de cualquier proceso de planeación que forme parte de la cosmovisión compartida o no por los integrantes de la sociedad. Desde esta perspectiva, se diseñan planes de desarrollo institucionales, que plasmen la realidad socioeconómica que la ideología del gobierno de turno asuma para desarrollar ese modo de pensar específico. 
En este orden de ideas, Díaz (2003, p. 1, citando la Asamblea Nacional, 2000) plantea que "el Plan de la Nación es el instrumento fundamental para organizar el desempeño de la acción del Estado Venezolano en función de alcanzar los grandes objetivos contenidos en la Constitución Nacional de la República Bolivariana de Venezuela".

El autor también comenta que "como herramienta de planificación es de imperativo cumplimiento para todas las instituciones, instancias y niveles de la administración pública centralizada y descentralizada; mientras que es sólo de carácter indicativo para el sector privado" (Díaz, 2003, p. 1).

Desde este punto de vista, el autor menciona que todas las instituciones del sector público, incluidas las universidades, deben adaptar sus planes y programas a los Lineamientos Generales del Plan de Desarrollo, Económico y Social de la Nación 2001-2007, para contribuir en sinergia al logro de los objetivos y metas establecidos en este documento.

Como puede verse, para el autor en este plan se parte de un diagnóstico de la situación del país, para luego caracterizar la realidad socio económica en cinco grandes dimensiones o desequilibrios (Díaz, 2003):

* Equilibrio económico: ...Basado en el crecimiento económico autosustentable, con diversificación productiva y competitividad internacional. ...

* Equilibrio social: Dirigido a alcanzar y profundizar el desarrollo humano, mediante la ampliación de las opciones de las personas, con mayores y mejores oportunidades efectivas de educación, salud, empleo, ingresos, organización social y seguridad ciudadana...

* Equilibrio político: Consolidar la estabilidad social, desarrollar el nuevo marco jurídico institucional y contribuir al establecimiento de la democracia participativa y protagónica.

* Equilibrio territorial: [Promover] modificaciones del patrón de poblamiento, producción, inversión, distribución y recaudación, cual se estima viable a mediano y a largo plazo.

* Equilibrio internacional: [Promueve] la integración latinoamericana y caribeña, avanzando hacia una comunidad de naciones, defendiendo los intereses económicos, sociales, culturales, políticos y ambientales de la Región. (p. 3)

Finalmente al autor afirma que "hay grandes desequilibrios en lo económico, lo social, lo político, lo territorial y lo internacional. Entonces el Plan se organiza en cinco dimensiones en las cuales hay que establecer equilibrios para enfrentar y corregir los desequilibrios" (Díaz, 2003, p. 3). 


\section{Marco metodológico}

La presente investigación asume el posicionamiento epistemológico empírico analítico o paradigma positivista, ya que se establecen conexiones hipotético-deductivas de enunciados que permiten la deducción de hipótesis empíricamente relacionadas con la finalidad de proponer un modelo de estructura organizacional para los institutos universitarios vinculante con la realidad socioeconómica venezolana.

También, este estudio reúne las características que permiten ubicarlo como una investigación que contempla la elaboración y desarrollo de un modelo, por lo cual se ubica dentro de la tipología proyectiva o modalidad proyecto factible, con apoyo en una investigación de campo de carácter descriptiva, con un diseño de campo no experimental, transeccional, debido a que mediante la aplicación de los instrumentos de recolección de información, como son los cuestionarios y las entrevistas, se pudo determinar el nivel de incidencia y el valor de la variable estudiada.

Por otra parte, esta investigación estuvo dirigida al subsistema de educación superior, específicamente a los institutos universitarios de tecnología de Maracaibo y Cabimas, ubicados geográficamente en las ciudades de Maracaibo y Cabimas del estado Zulia. Esta investigación se realizó durante el período comprendido entre los meses de junio a noviembre del año 2010.

La población estuvo conformada por un conjunto de 746 sujetos de investigación integrada por dos (02) directores, dos (02) subdirectores académicos, dos (02) subdirectores administrativos y 740 profesores. Para el estrato poblacional de directores, subdirectores administrativos y académicos se asumió como muestra la totalidad recurriendo a un censo. Para el caso del estrato poblacional profesores, se recurrió para su determinación la fórmula propuesta por Sierra (2001), citado en Pertuz, 2013), para poblaciones finitas, quedando definida como $n=88$. Para la determinación de la muestra en las diferentes instituciones se aplicó la fórmula de Shiffer (1997), citado por Sierra (2001), y ambos citados por (Pertuz, 2013), arrojando los resultados mostrados en la tabla 1 (Pertuz, 2013):

Tabla 1

Distribución de la muestra de profesores por instituciones

\begin{tabular}{lc}
\hline \multicolumn{1}{c}{ Instituciones } & Profesores \\
\hline Instituto Universitario de Tecnología de Maracaibo & 51 \\
Instituto Universitario de Tecnología de Cabimas & 37 \\
\hline Total & $\mathbf{8 8}$ \\
\hline
\end{tabular}

Nota. Departamento de Recursos Humanos de los institutos universitarios de tecnología. 
URL: http://www.una.ac.cr/educare

CORREO: educare@una.cr

Para la recolección de datos se diseñó un cuestionario de preguntas cerradas, con escala de Likert, utilizando cuatro alternativas de respuesta Nunca (1), Casi nunca (2), Casi siempre (3), Siempre (4). Este instrumento aplicado presentó cincuenta y cuatro (54) afirmaciones o ítems correspondientes a las variables analizadas.

De acuerdo con la escala de Likert mencionada, se determinaron las frecuencias de respuestas de acuerdo con los cuatro criterios utilizados en el instrumento aplicado, tomando como tendencia negativa las opciones (1) y (2), como la tendencia media las opciones (2) y (3), y finalmente la tendencia positiva las opciones (4) y (5) de la encuesta, para de esta forma considerar a toda la muestra por igual, sin dejar de evaluar a cada uno de las intenciones de respuestas generadas por la población encuestada.

Para la verificación de la validez del instrumento, se recurrió a la verificación de las afirmaciones o ítems a través de la consulta de cinco (5) expertos, tomándose en cuenta su estructura, redacción, vocabulario, tipo de preguntas y la pertinencia de los contenidos. En cuanto a la confiabilidad, se determinó aplicando una prueba piloto a una muestra de 15 sujetos con características similares a las del estudio y cuyos resultados se les aplico el coeficiente de confiabilidad de CRONBACH (a), con el apoyo del paquete estadístico SPSS for windows, versión 10.0, arrojando como resultado $a=0,9261$, lo cual comprueba un nivel aceptable de confiabilidad. (Pertuz, 2013, p. 13)

\section{Análisis y discusión de resultados}

Tomando en cuenta las opiniones recolectadas a través de los instrumentos, a continuación se presentan los resultados obtenidos de la aplicación del instrumento sobre la variable estructura organizacional, el cual fue suministrado a la población seleccionada para dar respuesta al objetivo dirigido a analizar la estructura organizacional de los institutos universitarios venezolanos.

En la tabla 2 se muestran los resultados de la dimensión tipo de estructura organizacional, obtenidos de los directores sub-directores administrativo, sub-directores académicos y profesores de los institutos universitarios de Maracaibo y Cabimas consultados en la muestra, los cuales indican la coexistencia de las tipologías estructurales siguientes: Las burocráticas profesional, mecánica y carismática, y las estructuras departamental y simple. 
Tabla 2

Dimensión tipo de estructura organizacional

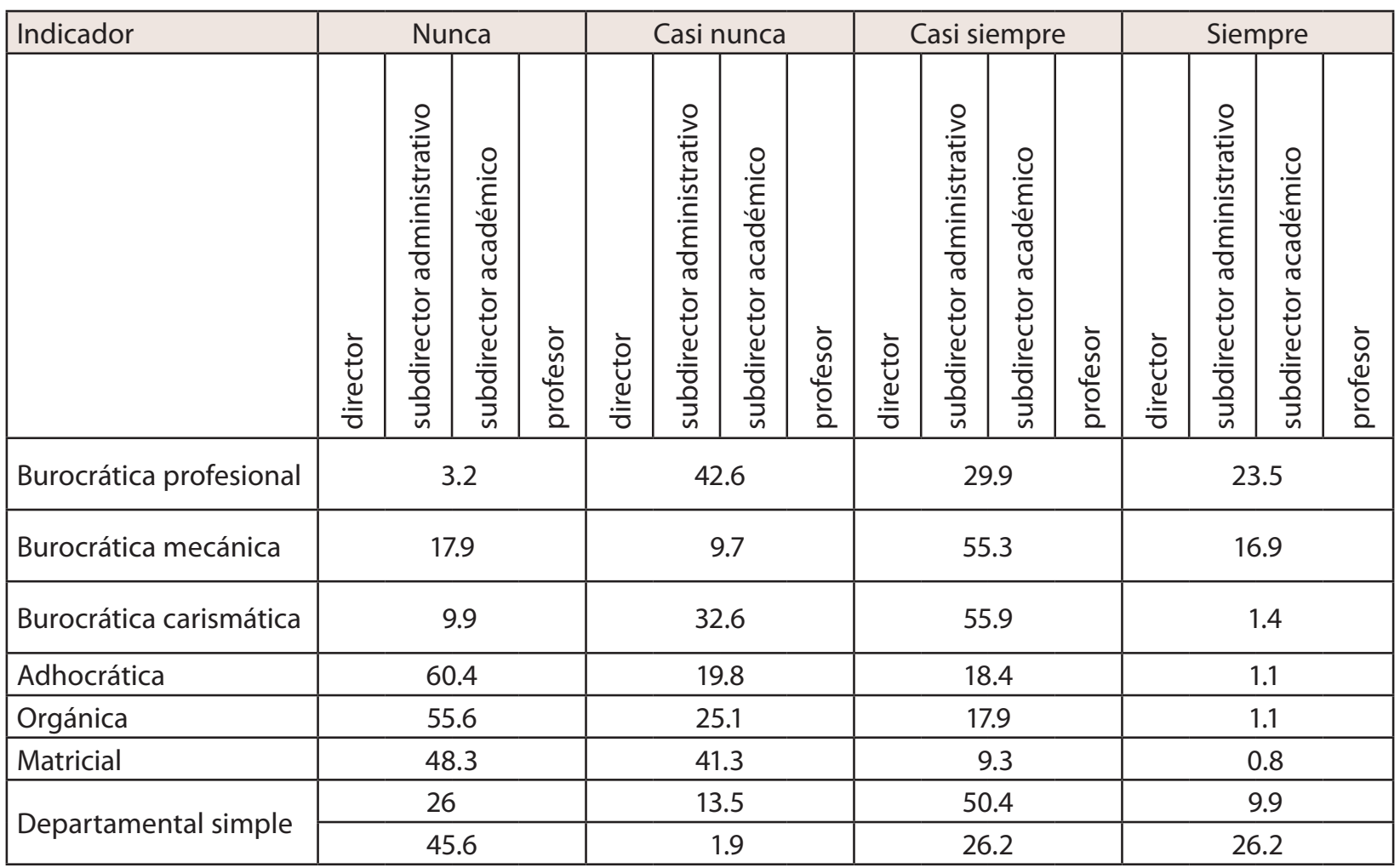

Nota. Elaboración propia.

Con respecto a lo antes planteado, puede evidenciarse en estas tipologías estructurales burocráticas presentes en los tecnológicos de Cabimas y Maracaibo, el predominio de los procesos académicos y administrativos por parte del personal de dirección, subdirección administrativo y académico, a través de un estricto cumplimiento de los procedimientos y normas establecidas, lo cual imposibilita la participación de los otros estratos presentes en estas organizaciones.

Para el logro de estos controles, los institutos universitarios analizados se apoyan en una estructura jerárquica, impersonal, fuertemente centralizada e inflexible, además de un liderazgo autocrático ejercido por los directivos, quienes sustentan el ejercicio del poder con una estructura altamente profesionalizada. 
URL: http://www.una.ac.cr/educare

CORREO: educare@una.cr

Seguidamente, en la tabla 3 se presentan los resultados obtenidos de la dimensión integración de los subsistemas, obtenidos del personal de dirección, subdirección administrativa, subdirección académica y profesorado de los institutos universitarios de Maracaibo y Cabimas consultado en la muestra. Estos resultados evidencian "una clara desintegración de los subsistemas integrantes de la estructura organizacional, por lo cual puede inferirse que el sistema y sus partes, es decir, los subsistemas, se encuentran en desequilibrio impidiendo así el cumplimiento de las funciones sustantivas de las instituciones analizadas, además de constituirse un obstáculo para el logro de las metas y objetivos institucionales". (Pertuz, 2013, Análisis y discusión de resultados, párr. 3)

Tabla 3

Dimensión integración de los subsistemas

\begin{tabular}{|c|c|c|c|c|c|c|c|c|c|c|c|c|c|c|c|c|}
\hline \multirow[t]{2}{*}{ Indicador } & \multicolumn{4}{|c|}{ Nunca } & \multicolumn{4}{|c|}{ Indicador } & \multicolumn{4}{|c|}{ Casi siempre } & \multicolumn{4}{|c|}{ Siempre } \\
\hline & $\begin{array}{l}\overline{\bar{U}} \\
\stackrel{.}{\stackrel{\Xi}{0}}\end{array}$ & 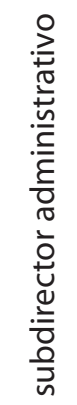 & 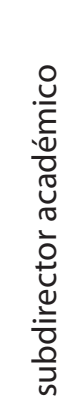 & 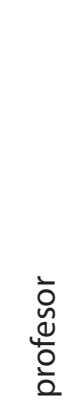 & $\begin{array}{l}\overline{\grave{U}} \\
\stackrel{\underline{\Xi}}{\overline{0}}\end{array}$ & 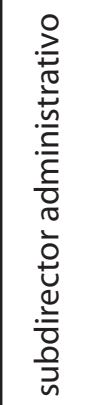 & 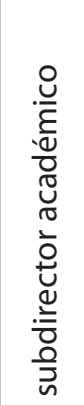 & 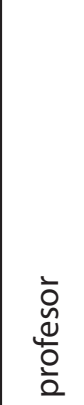 & $\begin{array}{l}\overline{\bar{U}} \\
\stackrel{\stackrel{d}{0}}{.0}\end{array}$ & 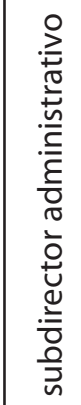 & 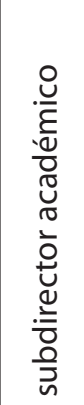 & $\begin{array}{l}\overline{0} \\
\stackrel{0}{0} \\
\frac{0}{0}\end{array}$ & 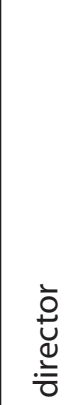 & 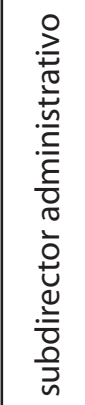 & 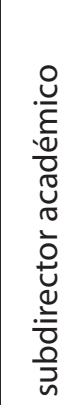 & 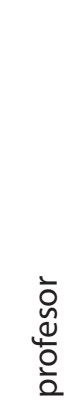 \\
\hline Ideológico & 66.6 & 33.3 & 33.3 & 58.3 & 33.3 & 33.3 & 33.3 & 33.7 & 0.0 & 33.3 & 33.3 & 4.5 & 0.0 & 0.0 & 0.0 & 3.4 \\
\hline $\begin{array}{l}\text { Orgánico- } \\
\text { estructural }\end{array}$ & 16.6 & 16.6 & 0.0 & 34.1 & 66.6 & 50 & 50 & 58 & 16.6 & 33.3 & 50 & 3.8 & 0.0 & 0.0 & 0.0 & 4.1 \\
\hline Tecnológico & 0.0 & 0.0 & 0.0 & 8.7 & 50 & 50 & 33.3 & 57.6 & 33.3 & 50 & 50 & 30 & 16.6 & 0.0 & 16.6 & 3.8 \\
\hline Psicosocial & 0.0 & 0.0 & 0.0 & 5.7 & 83.3 & 66.6 & 66.6 & 87.5 & 16.6 & 16.6 & 33.3 & 3.4 & 0.0 & 0.0 & 0.0 & 3.4 \\
\hline Normativo & 0.0 & 0.0 & 0.0 & 6.1 & 16.6 & 16.6 & 33.3 & 4.5 & 50 & 50 & 66.6 & 33.3 & 33.3 & 33.3 & 0.0 & 56.1 \\
\hline Promedio & 17 & 10 & 7 & 23 & 50 & 43.3 & 43.3 & 48.3 & 23.3 & 37 & 47 & 15 & 10 & 7 & 3.3 & 14.2 \\
\hline Porcentaje & \multicolumn{4}{|c|}{$14 \%$} & \multicolumn{4}{|c|}{$46 \%$} & \multicolumn{4}{|c|}{$31 \%$} & \multicolumn{4}{|c|}{$9 \%$} \\
\hline
\end{tabular}

Nota. Elaboración propia. 
En la tabla 4 se continúa con el análisis de la variable estructura organizacional, la cual arrojó el siguiente resultado: el 34.7 \% del personal de dirección, subdirección administrativa, subdirección académica y profesorado de los institutos universitarios de Maracaibo y Cabimas consultado en la muestra manifestaron que casi nunca, el $32 \%$ casi siempre, el $23.8 \%$ nunca y el $9,5 \%$ siempre. Estos resultados indican en la muestra seleccionada una tendencia negativa en cuanto a la estructura predominante en estas instituciones y a la integración de los subsistemas.

Tabla 4

Resultados obtenidos de la variable estructura organizacional

\begin{tabular}{|c|c|c|c|c|c|c|c|c|c|c|c|c|c|c|c|c|}
\hline \multirow{2}{*}{ Dimensión } & \multicolumn{4}{|c|}{ Nunca } & \multicolumn{4}{|c|}{ Casi nunca } & \multicolumn{4}{|c|}{ Casi siempre } & \multicolumn{4}{|c|}{ Siempre } \\
\hline & $\begin{array}{l}\overline{0} \\
\stackrel{ \pm}{0} \\
. \overline{0}\end{array}$ & 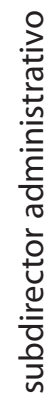 & 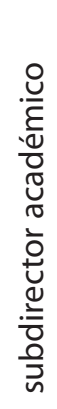 & 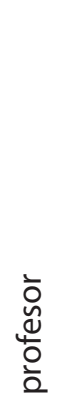 & 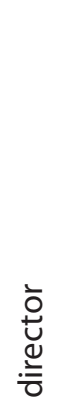 & 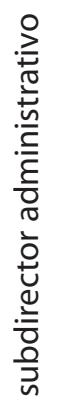 & 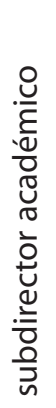 & 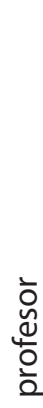 & 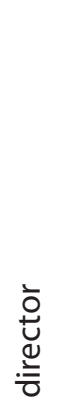 & 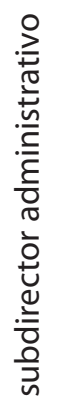 & 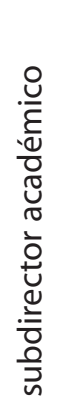 & $\begin{array}{l}\overline{0} \\
\stackrel{\mathscr{U}}{0} \\
\text { ¿o } \\
\end{array}$ & 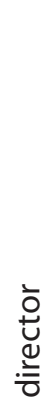 & 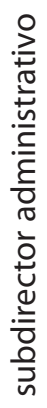 & 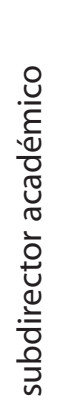 & 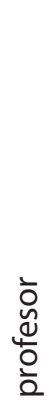 \\
\hline Tipos de estructura & \multicolumn{4}{|c|}{33.4} & \multicolumn{4}{|c|}{23.3} & \multicolumn{4}{|c|}{33} & \multicolumn{4}{|c|}{10.1} \\
\hline Integración de los subsistemas & \multicolumn{4}{|c|}{14.2} & \multicolumn{4}{|c|}{46.2} & \multicolumn{4}{|c|}{31} & \multicolumn{4}{|c|}{9} \\
\hline Porcentaje & \multicolumn{4}{|c|}{$23.8 \%$} & \multicolumn{4}{|c|}{$34.7 \%$} & \multicolumn{4}{|c|}{$32 \%$} & \multicolumn{4}{|c|}{$9.5 \%$} \\
\hline
\end{tabular}

Fuente. Elaboración propia.

Ahora bien, tomando en cuenta estos resultados, se debe mencionar que en la estructura organizacional presente en las instituciones analizadas no se evidencian la formalización de funciones ni el establecimiento de relaciones que determinan las misiones que cada unidad de la organización debe cumplir. Además, es perceptible un deterioro en los modos de colaboración entre estas unidades.

Por tanto, puede decirse que la estructura organizacional no se convierte en un elemento integrador de las actividades desarrolladas en las instituciones estudiadas, por lo cual puede decirse que la estructura organizacional en estas instituciones no presenta las funciones de elaborar productos organizacionales y alcanzar objetivos organizacionales, debido a la alta burocratización de la estructura.

Seguidamente, en la tabla 5, se continúa con el análisis de los resultados de la variable realidad socioeconómica, obtenidos de la aplicación del instrumento, el cual fue suministrado a la muestra seleccionada, para dar respuesta al objetivo dirigido a analizar la realidad socioeconómica de los institutos universitarios venezolanos. 
URL: http://www.una.ac.cr/educare

CORREO: educare@una.cr

Tabla 5

Resultados obtenidos de la variable realidad socioeconómica

\begin{tabular}{|c|c|c|c|c|c|c|c|c|c|c|c|c|c|c|c|c|}
\hline \multirow[t]{2}{*}{ Dimensión } & \multicolumn{4}{|c|}{ Nunca } & \multicolumn{4}{|c|}{ Casi nunca } & \multicolumn{4}{|c|}{ Casi siempre } & \multicolumn{4}{|c|}{ Siempre } \\
\hline & $\begin{array}{l}\grave{ \pm} \\
\stackrel{ \pm}{ \pm} \\
\stackrel{.}{0}\end{array}$ & 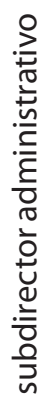 & 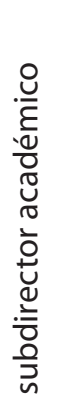 & 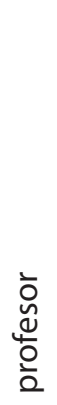 & 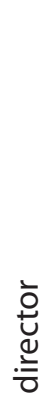 & 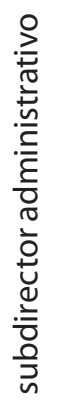 & 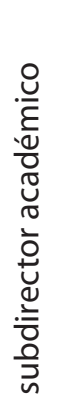 & 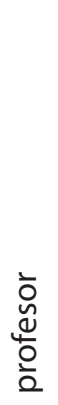 & 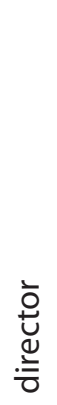 & 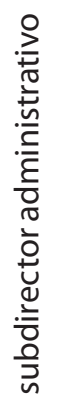 & 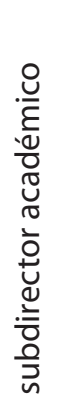 & 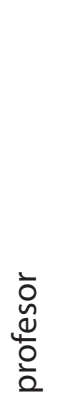 & 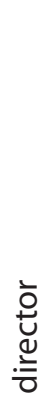 & 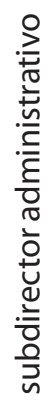 & 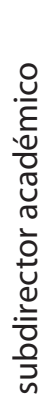 & 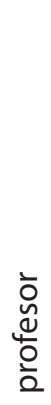 \\
\hline Realidad social & \multicolumn{4}{|c|}{16.6} & \multicolumn{4}{|c|}{45.2} & \multicolumn{4}{|c|}{28.1} & \multicolumn{4}{|c|}{9.6} \\
\hline Realidad económica & \multicolumn{4}{|c|}{24.8} & \multicolumn{4}{|c|}{45.4} & \multicolumn{4}{|c|}{20.5} & \multicolumn{4}{|c|}{9.1} \\
\hline Porcentaje & \multicolumn{4}{|c|}{$20.7 \%$} & \multicolumn{4}{|c|}{$45.3 \%$} & \multicolumn{4}{|c|}{$24.3 \%$} & \multicolumn{4}{|c|}{$9.3 \%$} \\
\hline
\end{tabular}

Nota. Elaboración propia.

El análisis de la variable de la realidad socioeconómica arrojó el siguiente resultado: el $45.3 \%$ de los directores, subdirectores administrativos, subdirectores académicos y profesores de los institutos universitarios de Maracaibo y Cabimas consultados en la muestra manifestaron que casi nunca, el $24.3 \%$ casi siempre, el $20.7 \%$ nunca y el $9.3 \%$ siempre. Estos resultados indican en la muestra seleccionada una tendencia negativa.

Como puede verse, estos resultados plantean claramente la descontextualización en la aplicación de los planes de la nación, en estas instituciones. Esto indica poca adecuación de sus planes organizacionales particulares y objetivos y metas no acordes con los planes de la Nación de la República Bolivariana de Venezuela.

Es por ello que, Díaz (2003), plantea que "aquellas universidades o unidades académicas particulares que no adecuen o actualicen su oferta académica no estarán atendiendo las nuevas demandas sociales, ni los requerimientos de nuevos segmentos de los mercados de trabajo (p. 3), lo cual niega la maximización del bienestar colectivo, expresado en ampliación de la democracia, mayor, seguridad social, crecientes fuentes de trabajo, alto valor agregado Nacional, mejor nivel de vida de la población y mayor soberanía del País (Díaz, 2003, p. 1).

\section{Propuesta}

Para establecer en estas instituciones que se encuentran en un modelo de estructura cerrado se transformen a otro abierto que brinde soporte a la organización, se hace necesario constituir redes académicas (unidades curriculares) interconectadas, a través de la incorporación de una estructura organizativa departamental, para luego implantar el modelo matricial. 
La conducción o migración de estructura departamental a matricial permitirá más flexibilidad curricular en la docencia, la interdisciplina en la investigación y la pertinencia social. Los modelos matriciales-departamentales suelen llamárseles modelos mixtos, ya que estos funcionan con mayor eficiencia en la consecución de los propósitos académicos de la organización. Sin embargo, constituyen un cambio radical respecto del modelo burocrático poco flexible e ineficiente que prevalece en los institutos tecnológicos universitarios.

Con este modelo se espera que la organización propicie el trabajo colegiado y la interdisciplina, vincule la investigación y la docencia, permita al estudiantado construir sus rutas de formación, se optimicen los recursos al ofrecerse cursos compartidos, y los programas y presupuestos se descentralicen. Asimismo, las estructuras matriciales-departamentales aseguran una mayor flexibilidad y capacidad de respuesta ante las necesidades cambiantes del entorno.

Sin embargo, debe aclararse que ningún modelo de organización puede garantizar la calidad y pertinencia de la vida académica: es su adaptación y construcción sobre la marcha la que irá dando el modelo funcional real. En este sentido, se puede concebir la departamentalización como un proceso que se irá asentando y mejorando en la práctica para percibir objetivamente sus beneficios. Siendo la departamentalización una implicación fundamentalmente académica, es necesario que este proceso se sustente en una amplia consulta y consenso principalmente con la planta de profesores de tiempo completo y con las autoridades relacionadas con las funciones sustantivas de docencia, investigación y extensión.

Para la aplicación de este modelo se hace necesaria su instrumentación por etapas que permitan alcanzar las características operativas y las bondades del modelo departamental, en concordancia con el marco institucional y el modelo educativo que postula el Ministerio de Educación Universitaria. Esta estrategia se definirá en tres momentos propuestos e implementados en la Universidad del Caribe, Quintana, (2003) citado por Pertuz (2007):

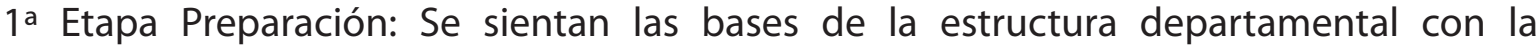
integración de cuerpos académicos disciplinarios. Se enfocará fundamentalmente a la docencia concretando la flexibilidad curricular, el sistema de créditos, delimitar el alcance de la institución por áreas de conocimiento y programas, perfiles, mecanismos de coordinación de las carreras, relaciones de gobierno y autoridad y capacitación de directivos académicos. Se constituirán unidades disciplinarias, que agrupen a todas las actividades necesarias para formar especialistas en varias áreas profesionales análogas. Se deberán realizar las siguientes actividades: a) Elaboración del proyecto, b) Aprobación del proyecto, c) Nombramiento de jefes de departamento, d) Capacitación de jefes de departamento, e) Adscripción de los Profesores a cada Departamento y f) Orientación a los profesores de cada departamento.

2a Etapa Operación: Orientada a los cuerpos académicos y la descentralización presupuestal, inicio de la operación departamental, programa-presupuesto descentralizado, reglamentación aprobada, registro de los cuerpos académicos, acceso a recursos por los cuerpos académicos y mecanismos de evaluación del trabajo académico. Se deberán realizar 
URL: http://www.una.ac.cr/educare

CORREO: educare@una.cr

las siguientes actividades: a) Determinar estructura interna de los departamentos, b) Análisis de los programas de las materias y los objetivos de los planes de estudio para determinar las áreas, c) Determinar los troncos comunes de cada área, con sus equivalencias en créditos, d) Establecer el sistema de créditos y e) Elaborar y revisar los programas y objetivos de las materias de los primeros semestres.

3a Etapa Consolidación: Orientada a la vinculación de la docencia y la investigación, programas de investigación y desarrollo tecnológico que deriven de proyectos departamentales, establecimiento de una Unidad de Gestión de Servicios Tecnológicos, consolidación de la planta docente por disciplina, reformas curriculares registradas ante profesiones, ampliación de oferta a partir de las interfases disciplinarias y mecanismos de autoevaluación departamental. Se deberán realizar las siguientes actividades: a) Determinar seriación de materias, b) Determinar los créditos correspondientes a los programas de los primeros semestres del tronco común, c) Elaboración de las cartas descriptivas de las materias del tronco común, d) Determinación de salidas terminales, e) Creación de nuevas carreras, f) Creación de salidas laterales y g) Proyectos de investigación.

Seguidamente, se definirán líneas de acción para impulsar el proceso de la departamentalización, basadas en la problemática presentada en el proceso, organizándolos en áreas claves, para su resolución: Cultura organizacional, carrera académica, vida colegiada, descentralización de la planeación operativa y marco reglamentario.

\section{Consideraciones del modelo de estructura organizacional}

Un modelo es una forma de representar una realidad determinada, por tanto, un ideal que permita mirar las complejidades desde perspectivas multivariadas. Desde esta idea, un modelo no debe ser entendido como la realidad total que representa, ya que, en cierta manera, involucra el uso de la racionalidad subjetiva y objetiva.

Así pues, el modelo de estructura organizacional propuesto en esta investigación integra las estructuras organizacionales surgidas en las organizaciones estudiadas, a través de los instrumentos aplicados, lo cual supone la migración de este pensamiento burocrático administrativo, a otro que proporcione flexibilidad en la forma de hacer las cosas en la organización.

Desde estas bases, la construcción de este modelo ideal de organización nunca podrá comprender toda la realidad, ya que como se ha dicho reiteradamente, solo se pretende acercarse a esta realidad compleja para adaptarse de forma eficiente; además, este podrá utilizarse para entender las relaciones e interacciones fundamentales que se desarrollan dentro de la organización, a fin de poder emprender acciones que permitan el desarrollo organizacional. 
De acuerdo con lo anterior, se propone un modelo mixto departamental/matricial de estructura que integre el departamento en una red matricial que incluye las actividades sustantivas de los institutos universitarios como son: docencia, investigación y extensión. El modelo propuesto intenta superar la visión de las estructuras organizacionales burocráticas profesionales rígidas e inflexibles. El modelo departamental-matricial propuesto supone la integración de las actividades sustantivas de docencia, investigación y extensión, a través de la unidad académica (UA) como eje de interacción estructural. Esta unidad estructural relacional básica puede observarse en la figura 1:

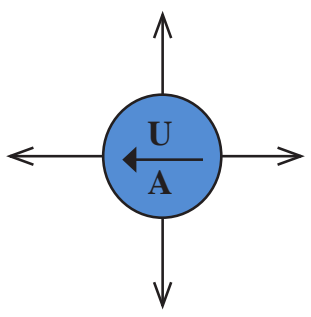

Figura 1. Unidad estructural relacional básica. Elaboración propia.

Como puede verse, las relaciones de integración corresponden a la agrupación de las actividades académicas docencia, investigación y extensión; además de la consolidación de una unidad de gestión, que promoverá la interacción administrativa, para la gestión de las necesidades y requerimientos para el desarrollo organizacional.

Visto lo anterior, la unidad estructural relacional básica asegura la transdisciplinariedad del conocimiento en la organización, a través del departamento como órgano colegiado (consejo departamental), desde donde se propicia la participación y el cambio organizacional.

Ahora bien, las unidades estructurales relacionales básicas, se integran con otras a través de los departamentos, lo cuales establecen relaciones con el modelo de carrera actual, que solo debe mirarse como de transición, ya que se espera con esta propuesta migrar la estructura de burocrática a departamental-matricial y, finalmente, matricial.

Visto de esta forma, los departamentos son integrados por unidades de gestión o mandos integrales que se encargan de hacer eficientes todos los procesos relacionales de la organización, que son a su vez discutidos en el consejo departamental donde finalmente son discutidos todos aquellos procesos que deben ejecutarse para el desarrollo organizacional. La propuesta de estructura puede visualizarse de la figura 2: 


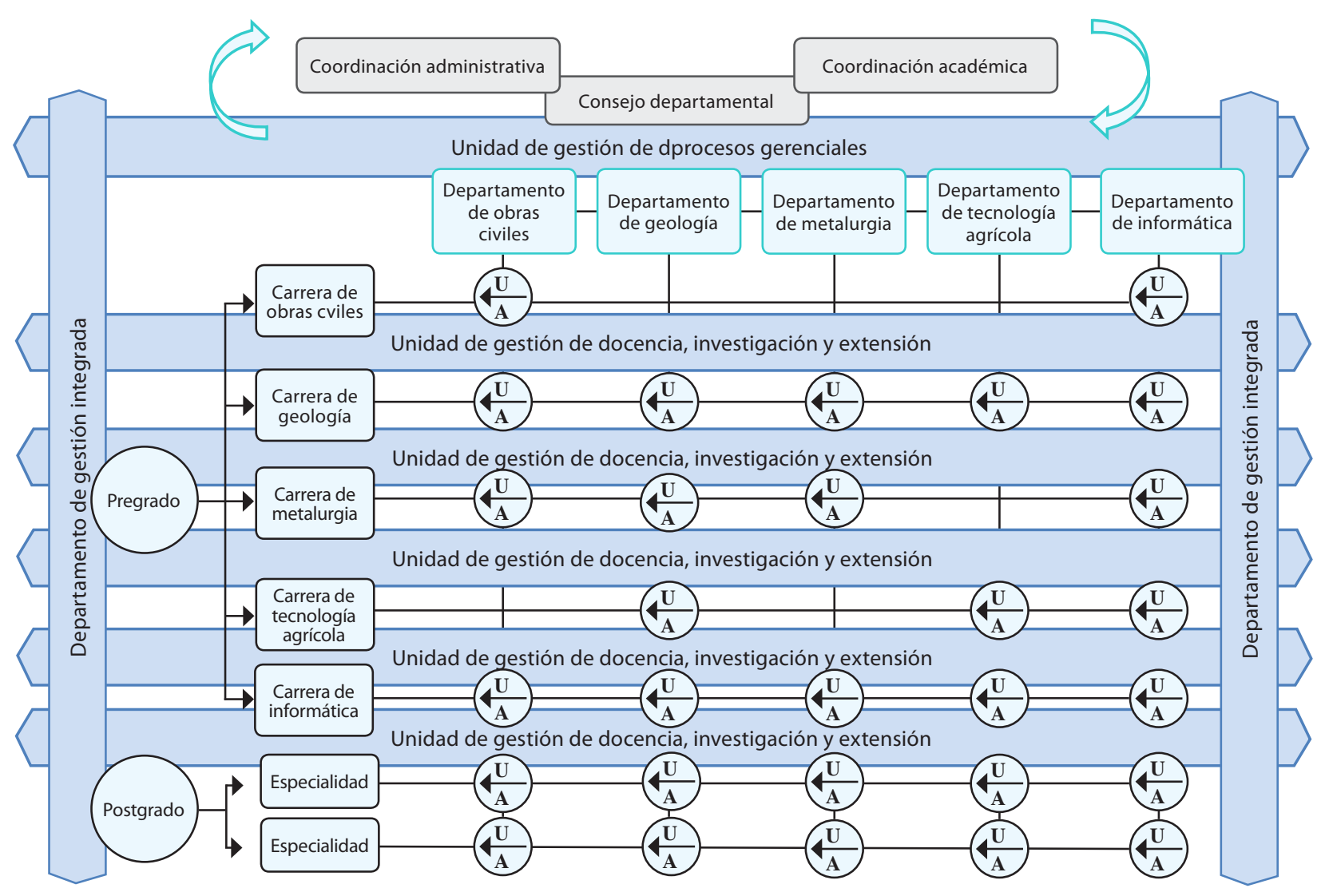

Figura 2. Propuesta de estructura organizacional. Elaboración propia.

\section{Evaluación de impacto}

La estructura departamental-matricial que en estas instituciones se pretenda implementar debe estar orientada a definir, en amplio sentido, su pertinencia socioeconómica, social e institucional, contenida en los planes de la nación, y establecer las orientaciones que permitan su acoplamiento orgánico.

Es obvio que debe considerarse esta estructura como dinámica y eficaz. Esta permitirá evitar la repetición de los defectos e insuficiencias que han caracterizado a estas instituciones de educación superior, cuya rigidez y desarticulación limita su eficacia y su alcance, así como su capacidad de enfrentarse con éxito a los procesos de cambio económico y social. Por ello, este modelo de estructura impactará a estas instituciones de la siguiente forma:

1) A nivel de docencia: Propiciará la flexibilidad curricular, con un enfoque centralizado en sus estudiantes como capital humano especializado; fortalecerá la figura del personal docente-investigativo en áreas acordes con la realidad socioeconómica imperante; 
ampliará la oferta académica por ofrecer en áreas interconectadas con las realidades del entorno e impulsará la creación de sistemas de tutorías en interdiciplinas.

2) A nivel de investigación: Propiciará la interdisciplinariedad a través de las unidades académicas presentes en las redes y cuerpos académicos, y fortalecerá la vinculación investigación-docencia.

3) A nivel de extensión: Logrará fortalecer las relaciones con las comunidades del entorno, propiciando la comunicación y mayor vinculación entre todos los integrantes de las comunidades cercanas a estas instituciones.

4) A nivel de gestión: Propiciará mayor vida académica colegiada, donde las decisiones gerenciales sean tomadas con la participación de todos los actores relacionados con la organización, se logrará mayor optimización de los recursos y, finalmente, se logrará la descentralización operativa y presupuestal.

\section{Conclusiones}

Esta propuesta toma en cuenta los resultados obtenidos de las opiniones recolectadas de los sujetos de los institutos universitarios de Maracaibo y Cabimas, consultados en la muestra, quienes manifestaron la coexistencia de las tipologías estructurales burocráticas profesional, mecánica y carismática, además de las estructuras departamental y simple.

Desde estas ideas, puede evidenciarse, en estas tipologías estructurales burocráticas presentes en los tecnológicos de Cabimas y Maracaibo, el predominio de los procesos académicos y administrativos, por parte del personal con cargos de dirección y subdirección administrativa y académica, a través de un estricto cumplimiento de los procedimientos y normas establecidas, lo cual imposibilita la participación de los otros estratos presentes en estas organizaciones.

Para el logro de estos controles, los institutos universitarios analizados se apoyan en una estructura jerárquica, impersonal, fuertemente centralizada e inflexible, además de un liderazgo autocrático ejercido por los directivos, quienes sustentan el ejercicio del poder con una estructura altamente profesionalizada.

En cuanto a la integración de los subsistemas se concluyó que los mismos no se encuentran integrados en las instituciones estudiadas, lo cual evidentemente genera y privilegia, en sus integrantes, patrones de comportamiento no acordes con la estructura y sus procesos. Es válido mencionar que estos comportamientos se encuentran alejados de la racionalidad, la flexibilidad y la creatividad, tan necesarias y exigidas por el modelo económico actual.

Desde este punto de vista, puede decirse que la estructura organizacional no se convierte en un elemento integrador de las actividades que se desarrollan en las instituciones estudiadas, por lo cual puede decirse que en la estructura organizacional de estas instituciones 
URL: http://www.una.ac.cr/educare

CORREO: educare@una.cr

no se presentan las funciones de elaborar productos organizacionales y alcanzar objetivos organizacionales, debido a su alta burocratización.

En lo referente al objetivo, dirigido a describir la realidad socioeconómica de los institutos universitarios venezolanos, se concluyó que los resultados obtenidos indican en la muestra seleccionada una tendencia negativa, lo cual plantea que dichas instituciones no son pertinentes con la realidad socioeconómica, ya que no satisfacen las demandas de la sociedad, pues no están vinculadas con los programas de desarrollo económico, social, cultural y regional ni con la consolidación de la estabilidad política y social, por lo cual no contribuyen al establecimiento de la democracia participativa y protagónica. Es decir, que las funciones de docencia, investigación y extensión, contenidas en los programas y actividades, no satisfacen el desarrollo de una educación de calidad para todas las personas ni tienen pertinencia social, así como tampoco alcanzan un crecimiento económico sostenido, ni eliminan la volatilidad económica, ni desarrollan la economía social, ni contribuyen al establecimiento de programas de capacitación específicos para la región, en la promoción de la democracia participativa y protagónica. Tampoco, lo hacen en la promoción y protección de los derechos humanos, ni en el apoyo de resolución pacífica de los conflictos en la región.

Por ello, estas instituciones no están atendiendo las nuevas demandas sociales, ni los requerimientos de nuevos segmentos de los mercados de trabajo, lo cual niega la maximización del bienestar colectivo, expresado en ampliación de la democracia, mayor seguridad social, crecientes fuentes de trabajo, alto valor agregado nacional, mejor nivel de vida de la población y mayor soberanía del país.

Finalmente, para el objetivo dirigido a proponer un modelo de estructura organizacional para los institutos universitarios vinculante con la realidad socioeconómica venezolana, se diseñó un modelo mixto departamental/matricial de estructura organizacional, que integra el departamento en una red matricial que incluye las actividades sustantivas de los institutos universitarios como son: docencia, investigación y extensión.

Con este modelo se espera que la organización propicie el trabajo colegiado y la interdisciplina, vincule la investigación y la docencia, permita a los estudiantes construir sus rutas de formación, se optimicen los recursos al ofrecerse cursos compartidos y los programas y presupuestos se descentralicen. Asimismo, que las estructuras matriciales-departamentales aseguren una mayor flexibilidad y capacidad de respuesta ante las necesidades cambiantes del entorno.

\section{Referencias}

Anzaldo, B. (enero-marzo, 1989). Estructura y organización académico-administrativa de las instituciones de educación superior. Revista de la Educación Superior, 18(69), 1-11. Recuperado de http://publicaciones.anuies.mx/revista/69/2/3/es/estructura-yorganizacion-academico-administrativa-de-las 
Brull, E. y Gil, M. (2005). Mintzberg: La estructuración de las organizaciones. Cuadernos de Gestión, 3, 1-20. Recuperado de https://www.u-cursos.cl/facso/2010/1/PSI-PTO/1/material docente/objeto/560068

Cardoso, E. O. (2006). Evaluación de la organización académico-administrativa de tres programas de maestría en el campo de la educación con relación a parámetros del CONACYT (Tesis doctoral). Instituto Politécnico Nacional. Escuela superior de comercio y administración, México.

Castellano, M. E. (noviembre, 2002). Institutos universitarios tecnológicos y colegios universitarios en Venezuela. Trabajo presentado en el 3er encuentro Europa-América Latina sobre formación tecnológica y profesional. La Habana, Cuba. Recuperado de http://www.univparis-diderot.fr/comm/infodoc/cdrom1/Conferencias/1\%20U\%20T\%20VENEZUELA.pdf

Daft, R. L. (2000). Teoría y diseño organizacional (6a ed.). México: Editorial Thomson Internacional.

Díaz, B. (diciembre, 2003). La universidad y el plan de la Nación 2001-2007. Academia, 2(3), 1-6. Recuperado de http://www.saber.ula.ve/bitstream/123456789/16890/1/benito diaz.pdf

Espinoza, R. (enero-abril, 2009). El discurso ideológico-político en la concepción del desarrollo económico-social en lo regional en Venezuela. Multiciencias 9(1), 13-23. Recuperado http://www.redalyc.org/pdf/904/90411683003.pdf

Perturz, R. A. (2007). Estructura organizacional de los institutos universitarios vinculante con la realidad socio-económica venezolana (Tesis de Maestría). Universidad Rafael Urdaneta. Maracaibo, Venezuela.

Pertuz, R. A. (2013). Integración de los subsistemas de la estructura organizacional de los institutos universitarios venezolanos. REICE, 11(1). Recuperado de http://www.rinace.net/ reice/numeros/arts/vol11num1/art1 htm.html

Toribio, D. (1999). La evaluación de la estructura académica. Buenos Aires: CONEAU. Recuperado de http://www.coneau.gob.ar/archivos/publicaciones/estudios/toribio.pdf

\section{Cómo citar este artículo en APA:}

Pertuz, R. (enero-abril, 2014). Modelo de estructura organizacional para los institutos universitarios, vinculante con la realidad socioeconómica venezolana. Revista Electrónica Educare, 18(1), 97-117. Recuperado de http://www.revistas.una.ac.cr/index.php/EDUCARE/issue/current

Nota: Para citar este artículo en otros sistemas puede consultar el hipervínculo "Como citar el artículo" en la barra derecha de nuestro sitio web:

http://www.revistas.una.ac.cr/index.php/EDUCARE/index 